\title{
HONEY, AN ADJUVANT THERAPY IN ACUTE INFANTILE DIARRHEA
}

\author{
H. Elnady ${ }^{1}$, N.A.A. $\mathrm{Aly}^{1}$, N.A. El Hussieny ${ }^{2}$, S. Kholoussi ${ }^{3}$ \\ ${ }^{I}$ Child Health Department, National Research Centre, ${ }^{2}$ General Organisation of Teaching Hospitals and \\ Institute, ${ }^{3}$ Immunogenetics, National Research Centre, Cairo, Egypt
}

Rationale or background: Diarrheal diseases is one of the major causes of mortality of infants and toddlers in developing countries ${ }^{(1)}$. Honey has an antimicrobial and anti-inflammatory effect so we aim to study the effect of using pure honey as an adjuvant therapy to the oral rehydration solution in management of acute infantile diarrhea.

Methodology: The effect of floral honey on 200 patient aged 6-24 months, suffering from acute diarrhea (more than 3 liquid motions and duration $\leq 72$ hours) ${ }^{(2)}$ with mild to moderate dehydration. They were divided into a control group and three honey treated groups (50 patients each). The control group received the WHO-ORS only. The other three groups received floral honey in various forms:

The studied groups were observed for rehydration time, weight gain, amount of rehydration solutions consumed, vomiting, diarrhea and recovery time. Stool culture was done at admission, stool $\mathrm{pH}$, serum sodium and potassium

Results: In the pure honey-ORS group, the recovery time was $3.5 \pm 2.88$ days compared to $5.6 \pm 3.8$ days in the control group and 5.4 $\pm 3.7,5.3 \pm 3.8$ days in group I and III respectively, showing significant shorter recovery time $(\mathrm{P}<0.05)$ specially in cases of bacterial diarrhea. Also persistent diarrhea was significantly less in pure honey-ORS treated group than other groups $(\mathrm{P}<0.05)$

Conclusion: Honey is a non allergic, natural agent of high nutrient value. We observed a significant decrease in stool motions number and improvement of stool consistency in Acute infantile diarrhea treated with pure honey in addition to oral rehydration solution. 